\section{Birgitt Holschuh-Lorang}

Die Psychokardiologie gilt aus universitärer Sicht als bisher recht stiefmütterlich behandelter, jedoch vielversprechender medizinischer Zweig. Dass die «Nöte der Seele» das Herz beeinflussen könnten, galt vor 10 Jahren aus konservativer kardiologischer Sicht noch als Hokuspokus. Wenn man bedenkt, dass anamnestisch nur $50 \%$ der Patienten mit kardialen Beschwerden Risikofaktoren wie Hypertonie, Diabetes, Übergewicht, Hyperlipidämie, Bewegungsmangel usw. zeigen, liegt es nahe, weitere mögliche Ursachen in das Blickfeld zu rücken.

Bereits in der Antike waren $\mathrm{Zu}$ sammenhänge zwischen seelischen Belastungen und Herzerkrankungen bekannt. Auch der Volksmund hat diesbezügliche Zusammenhänge schon längst formuliert: «Ich habe es mir sehr zu Herzen genommen", «Da ist mir vor Schreck das Herz stehengeblieben» oder «Es hat mir das Herz gebrochen».

Die Psychokardiologie erforscht die Einflussnahme psychischer und psychosozialer Faktoren auf die Entwicklung kardialer Erkrankungen. Mittlerweile existieren weltweit 22 Arbeiten mit weit über 50000 untersuchten Probanden, die die Bedeutung von negativer Affektivität auf die Vorhersage von allgemeiner Mortalität und der koronaren Herzerkrankung bestimmt haben. Folgende negative Affekte spielen hierbei eine besondere Rolle:

1. Depression

2. Trauer

3. Ärger

4. Feindseligkeit

5. Ängste

6. Stress

7. Mobbing

8. Burn-out

\title{
Psychokardiologie - Erfahrungen mit Psychosomatischer Energetik
}

Nicht umsonst werden Depression, Trauer, Ärger und Feindseligkeit zuerst erwähnt, denn sie betreffen die sogenannte Typ-D-Persönlichkeit, die bezüglich kardialer Erkrankungen besonders gefährdet erscheint. Es handelt sich um Menschen mit beruflich hohen Anforderungen und geringem Entscheidungsspielraum, die zu Depressivität, Disstress, Unglücklichsein und Kummer neigen sowie ein fehlendes Selbstvertrauen haben, weil sie alles hinunterschlucken und in sich hineinfressen - der Typ des nach innen gekehrten Verlierers. Im Gegensatz dazu stehen die Typ-A-Persönlichkeiten ständig unter Strom, sind in dauernder Zeitnot und als sogenannte extrovertierte Kämpfertypen geprägt von Ehrgeiz, Hektik und Rivalität.

Untersuchungen haben ergeben, dass die negative Affektivität zu einer chronischen Stressbelastung mit einer Dauerausschüttung von Adrenalin, Noradrenalin und Cortisol führt und ferner den Dopamin- und Serotoninspiegel ungünstig beeinflusst. Es existieren Theorien, die besagen, dass es dadurch zu einer Lähmung von Herzmuskelzellen oder zu einer Verkrampfung von Koronararterien kommen kann. Demzufolge klagen die Patienten über thorakalen Druck oder über Herzrhythmusstörungen, oder aber eine koronare Herzkrankheit (KHK) oder ein abgelaufener Myokardinfarkt ist bereits nachweisbar.

Die übliche psychokardiologische oder kardiopsychosomatische Behandlung besteht aus psychotherapeutischen Gesprächen, Verhaltenstherapien, sozialmedizinischen Massnahmen, Compliance-Förderung und gegebenenfalls Krisenintervention, um bei betroffenen Patienten ein Um- denken, eine Verhaltensänderung und eine Entspannung zu erzielen. Nicht immer führt diese Vorgehensweise zum Ziel. Aus diesem Grund verwende ich in meiner Praxis seit vielen Jahren eine komplementärmedizinische Methode: die Psychosomatische Energetik (PSE).

Nach den Erfahrungen der PSE ist für eine stabile Psyche und ein körperliches Wohlbefinden ein weiteres Element mitverantwortlich. Es ist der Aspekt der Lebensenergie oder Energetik, der im Zusammenspiel von Psyche und Soma einen entscheidenden Faktor darstellt. Die Theorie der PSE besagt, dass eine ausreichende Aufnahme von Lebensenergie sowohl das autonome Nervensystem und damit die zelluläre Funktion steuert als auch für eine ausreichende psychische Stabilität verantwortlich ist. Die Methode der PSE - vom allgemeinärztlichen Kollegen Reimar Banis entwickelt erlaubt mithilfe eines Gerätes, mit dem man die verschiedenen EnergieEbenen ansteigend belastet (Reba ${ }^{\circledR}{ }_{-}$ Testgerät), den energetischen Zustand eines Patienten mittels Prozentzahlen zu erfassen. Zur Energietestung verwende ich den kinesiologischen Armlängentest (Abb. 1). Mit den erhaltenen Energiewerten kann man eine Aussage über die jeweilige körperliche Vitalität und seelische Belastbarkeit des Patienten treffen. In der Regel spiegeln die Messwerte die vom Patienten gefühlte Befindlichkeit, decken sich aber auch mit dem klinischen Befund.

Bei kranken Patienten finden sich normalerweise erniedrigte Messwerte. Die Ursache hierfür liegt erfahrungsgemäss in verdrängten emotionalen Konflikten, deren Konfliktinhalte durch das Testen thematisiert

\section{KARGER}

Fax +497614520714 Information@Karger.com www.karger.com
Dr. med. Birgitt Holschuh-Lorang

Fachärztin für Allgemeinmedizin-NHV

Zum Scheidberg 9, 66798 Wallerfangen-Gisingen, Deutschland 


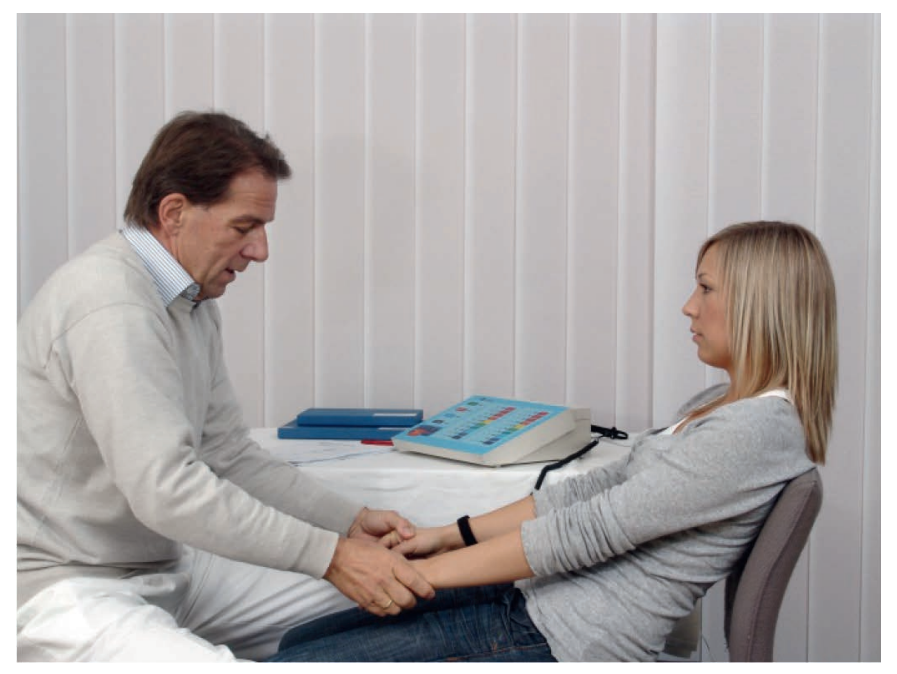

Abb. 1.

Kinesiologischer Armlängentest mithilfe des RebaTestgerätes.

werden können. Diese ins Unbewusste verdrängten Konflikte haben nicht nur Lebensenergie weggespeichert, sondern blockieren auch die Energieaufnahme. Mit speziellen homöopathischen Komplexmitteln können sie gelöst werden. Patienten erfahren durch diese Behandlung in der Regel einen Energiezuwachs, der wiederum das vegetative Steuerungssystem positiv beeinflusst und gleichzeitig die emotionale Belastbarkeit stärkt. In der Folge bessern sich oder verschwinden beklagte Beschwerden.

\section{Fallbeispiele}

Anhand von drei Patientenbeispielen soll die Wirkweise der Methode dargestellt werden.

\section{Fall 1: Peter, 52 Jahre}

Anamnese: Der Patient leidet seit Jahren an pektanginösen Beschwerden. Eine KHK ist nicht nachweisbar. Es besteht eine Hypertonie. Der Patient fühlt sich sehr erschöpft und neigt zu Depressionen. Mehrere Aufenthalte in psychosomatischen Kliniken erbrachten keinen Erfolg. Er klagt über Schlafstörungen mit «Stressträumen». Die derzeitige Behandlung erfolgt mit dem Antidepressivum Saroten ${ }^{\circledR}$, ohne dass eine merkliche Besserung erzielt werden konnte.
Bei der ersten Vorstellung zeigt die PSE-Diagnostik deutlich eingeschränkte Werte für die Vitalität (25\%; Sollwert $100 \%)$ und für die emotionale Belastbarkeit (20\%; Sollwert $100 \%$ ). Es besteht als äussere Energieblockade zusätzlich eine geopathische Belastung, die in der Regel einen Hinweis auf einen erdstrahlbelasteten Bettplatz gibt. In vier Therapiesitzungen können vier verschiedene unbewusste Konfliktthemen thematisiert werden. Es handelt sich um Themen wie verdrängte «Verängstigung», «inneres Angespanntsein», «Frust und Unzufriedenheit» und das Gefühl, «Opfer der Umstände» zu sein. Nach und nach werden diese Konfliktthemen mit den homöopathischen Komplexmitteln Chavita ${ }^{\circledR} 4 /$ Emvita $^{\circledR}$ 15, Chavita ${ }^{\circledR}$ 6/Emvita ${ }^{\circledR}$ 23, Chavita $^{\circledR} 3 /$ Emvita $^{\circledR} 11$ und Chavita ${ }^{\circledR} 5 /$ Emvita $^{\circledR} 18$ gelöst. Bei der letzten Untersuchung sind die Werte für die vitale Energie und die seelische Belastbarkeit auf jeweils 95\% gestiegen. Der Patient fühlt sich deutlich stabilisiert. Es geht ihm gut. Er hat keine pektanginösen Beschwerden mehr. Seinen Bettplatz hat er verstellt und er schläft gut. Saroten ${ }^{\circledR}$ wird nicht mehr benötigt.

\section{Fall 2: Christa, 49 Jahre}

Anamnese: Die Patientin klagt seit Monaten über retrosternalen Druck; sie spürt ständig einen «Kloss» in der Brust. Es besteht kein Anhalt für eine KHK. Sie lebt in einer schwierigen familiären Konfliktkonstellation, aufgrund derer sie sich ständig erschöpft und überfordert fühlt. Bei der ersten Messung sind die Werte für die vitale Energie mit 25\% (Sollwert 100\%) und für die emotionale Energie mit $20 \%$ (Sollwert 100\%) deutlich erniedrigt. Die getesteten Konfliktthemen «Panik», «Wut» und «Das Leben als Kampf erleben» werden mit den Komplexmitteln Chavita $^{\circledR} 4 /$ Emvita $^{\circledR} 16$, Chavita $^{\circledR} 3 /$ Emvita $^{\circledR} 9$ und Chavita ${ }^{\circledR}$ 7/Emvita ${ }^{\circledR} 26$ energetisch gelöst. Nach drei Therapiesitzungen sind die Energiewerte auf $75 \%$ vitaler Kraft und $70 \%$ emotionaler Energie gestiegen. Es geht ihr sehr viel besser, das retrosternale «Klossgefühl» ist verschwunden, ihre innere Haltung ist gestärkt und sie fühlt sich der familiären und beruflichen Situation viel besser gewachsen.

\section{Fall 3: Wolfgang, 54 Jahre}

Anamnese: Der Patient klagt häufig über kardiale Beschwerden wie Druckgefühl und Herzklopfen. Es bestehen ein Vorhofflimmern und ein Zustand nach Mitralklappenersatz. Eine Kardioversion ist erfolgt. Die von der Klinik verordneten Medikamente wie Digitoxin, Furosemid, Enalapril ${ }^{\circledR}$ und Concor ${ }^{\circledR}$ nimmt er nicht, da er sie nicht verträgt. Er fühlt sich müde und antriebsarm; als Lehrer ist er in seinem Beruf ständig angespannt.

Bei der Erstuntersuchung findet sich ein Vitalwert von $45 \%$ und ein Emotionalwert von 30\% - Werte, die seinem körperlichen und seelischen Befinden entsprechen. Die zu lösenden Konfliktthemen «Das Leben als Kampf erleben», «Panik», "Angespanntsein» und «Wut» werden mit Chavita $^{\circledR}$ 7/Emvita ${ }^{\circledR}$ 26, Chavita ${ }^{\circledR} 4 /$ Emvita $^{\circledR} 16$, Chavita ${ }^{\circledR}$ 6/Emvita ${ }^{\circledR} 23$ und Chavita ${ }^{\circledR} 3 /$ Emvita $^{\circledR} 9$ behandelt. Nach vier Therapiesitzungen hat auch er einen deutlichen Energiezuwachs erfahren - mit einem zuletzt gemesse- 
nen Wert von $90 \%$ für die vitale Energie und von $85 \%$ für die psychische Belastbarkeit. Er fühlt sich gut. Müdigkeit und Antriebsarmut sind deutlich gebessert. Das Herz macht ihm keine Probleme, und seinen Alltagsanforderungen kann er viel besser gerecht werden.

\section{Schlussfolgerung}

Wie sehr verdrängte Konflikte die Lebensenergie gewissermassen «wegvampirisiert» haben und das Herz in «Nöte» bringen können, haben die angeführten Fallbeispiele gezeigt. Dass es mithilfe des Testverfahrens der PSE möglich ist, diese Konfliktthemen zu testen und zu therapieren, hat in zahlreichen Fällen die kardialen
Probleme von Patienten verbessert oder sie gar von ihnen befreit. Gleichzeitig hat sich auch ihr seelisches Befinden stabilisiert.

Somit ist die PSE in ihrem wahrhaft ganzheitsmedizinischen Ansatz ein probates Test- und Therapieverfahren, das aus meinem medizinischen Alltag nicht mehr wegzudenken ist.

\section{Literatur}

Banis R: Durch Energieheilung zu neuem Leben. Petersberg, Via Nova, 2002.

Banis R: Lehrbuch der Psychosomatischen Energetik. VAK, Kirchzarten 2003.

Banis R (Hrsg): Lesebuch der Psychosomatischen Energetik. Band 1. Hochheim, Comed, 2004.

Banis R: Psychosomatische Energetik. Lehrbuch für Therapeuten. Kirchzarten, VAK, 2005.
Banis R (Hrsg): Lesebuch der Psychosomatischen Energetik. Band 2. Hoch heim, Comed, 2006.

Banis R (Hrsg): Lesebuch der Psychosomatischen Energetik. Band 3. Hochheim, Comed, 2007.

Banis R (Hrsg): Lesebuch der Psychosomatischen Energetik. Band 4. Hochheim, Comed, 2008.

Banis R: Multizentrische Praxisstudie zur Psychosomatischen Energetik. Schweiz Z Ganzheitsmed 2010;22:269-272.

Herrmann-Lingen C, Albus C, Titscher G: Psychokardiologie: Ein Praxisleitfaden für Ärzte und Psychologen. Köln, Deutscher Ärzte-Verlag, 2007.

Holschuh-Lorang B: Psychosomatische Energetik in der Allgemeinmedizin - Ergebnisse einer Praxisstudie. Schweiz Z Ganzheitsmed 2004; $16: 173-178$

Jordan J, Bardé B, Zeiher AM (Hrsg): Contributions toward Evidence-Based Psychocardiology: A Systematic Review of the Literature. Washington, DC, American Psychological Association, 2006.

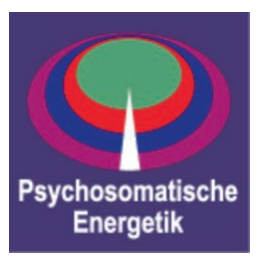

\section{Informationen zur Psychosomatischen Energetik}

Die seit 1997 etablierte Psychosomatische Energetik (PSE) ist dabei, zu einer Standardmethode der modernen Komplementärmedizin zu werden. Sie verbindet uralte Methoden wie den Schamanismus und das indische Yoga mit Psychoanalyse und Homöopathie zu einem neuen Ganzen. Ihre Grundüberlegung geht von der Erfahrung aus, dass ein harmonisches, von seelischen Traumen freies Energiesystem für die seelische und körperliche Gesundheit wichtig ist und eine entsprechende Therapie ganzheitliche Selbstheilprozesse in Gang setzt.

Die Ausbildung zum «zertifizierten Energietherapeut» verläuft berufsbegleitend und setzt sich aus mehreren festgelegten Ausbildungsabschnitten zusammen (Seminardaten siehe www.igpse.ch). Mittlerweile gibt es annähernd 500 ausgebildete Therapeuten im deutschsprachigen Raum. Die PSE wird darüber hinaus auch in Nordamerika sowie weiteren Ländern unterrichtet und mittlerweile in mehr als 20 Ländern weltweit praktiziert. Infomaterial für Therapeuten mit Hinweisen zur Ausbildung kann bei der Rubimed AG, Grossmatt 3, 6052 Hergiswil/Schweiz (www.rubimed.com) kostenlos angefordert werden. 